\section{Verb Semantic Structures in Memory for Sentences: Evidence for Componential Representation}

Dedre Gentner

Bolt Beranek and New'man, Inc., Cambridge, Massachusetts

This research contrasts two hypotheses concerning componential storage of meaning. The Complexity Hypothesis assumed by Fodor (The language of thought, NY: Crowell, 1975), Kintsch (The representation of meaning in memory, Hillsdale, NJ: Erlbaum, 1974), and Thorndyke (Conceptual complexity and imagery in comprehension and memory. Journal of Verbal Learning and Verbal Be havior, 1975, 14, 359-369) states that a word with many semantic components will require more processing resources, comprehension time, and long-term memory space than a word with few components, and thus will interfere more with memory for surrounding words. This memory prediction was tested against an alternative prediction based on connectivity. The Connectivity Hypothesis views verb semantic structures as frames for sentence representation and states that underlying verb subpredicates that connect the nouns. Thus, the Complexity Hypothesis predicts that a verb with many subpredicates will lead to poorer memory strength between the surrounding nouns than a verb with few subpredicates, while the Connectivity Hypothesis predicts that verbs with many subpredicates will lead to greater memory strength between nouns in cases when the additional subpredicates provide semantic connections between the nouns.

In three experiments, subjects recalled subject-verb-object sentences, given subject nouns as cues. General verbs, with relatively few subpredicates, were compared with more specific verbs whose additional subpredicates either did or did not provide additional connections between the surrounding nouns. The level of recall of the object noun, given the subject noun as cue, was predicted by the relative number of connecting subpredicates in the verb, but not by the relative umber of subpredic the Complexity Hypothesis. These results are interpreted in terms of a model in which the verb conveys a structured set of subpredicates that provides a connec tive framework for sentence memory.

The idea that word meanings have componential structures has a long history in western thought. The analysis of concepts into more basic concepts has been a tradition in philosophy at least since Socrates and Aristotle. More recently, componential representation has been an important theoretical idea for anthropologists (e.g., Romney \& d'Andrade,

The preparation of this paper was supported in part by The National Institute of Education under Contract No. US-NIE-C-400-76-0016. Some of the research described was carried out at the Psychology Department of the University of Washington and supported by departmental and university funds. I thank Philip Cohen, Allan Collins, Jerry Fodor, Ed Smith and Albert Stevens for their very helpful comments on earlier drafts of this paper. I also thank Erik Svehaug and Brenda Starr for their help in conducting the research, and Jill $O$ 'Brien for her inestimable help in preparing the manuscript. Send reprint requests to: Dedre Gentner, Department of Psychology, University of California, San Diego, La Jolla. CA 92093.
1964), linguists (e.g., Bendix, 1971; Bierwisch, 1971; Chafe, 1970; Fillmore, 1971; Lakoff, 1970; McCawley, 1968; Talmy, Note 4), computer scientists (e.g., Schank, 1972; Schank \& Colby, 1973; Schank, Goldman Rieger, \& Riesbeck, Note 5), and psychologists (e.g., Abrahamson, 1975; Clark, 1973; Clark, 1974; Genter, 1975; Rumelhart \& Levin, 1975; Smith, Shoben, \& Rips, 1974). It is easy to understand the appeal of the componential approach. It offers a powerful way of capturing generalities about relatedness among meanings, as well as a natural set of explanatory principles for some important psycholinguistic phenomena.

One example of this economy of explanation is the assumption that the degree of synonymity between words reflects the degree of overlap in their componential representations. This allows subjective similarity in meaning, substitutability in paraphrase, and confusability in long-term memory to be accounted for within one framework. Gentner (Note 2) measured the amount of shared substructure in the hypothesized componential representations for pairs of verbs. The degree of semantic overlap correlated highly with two empirical measures: (1) the degree to which the verb pairs were confused with one another in sentence recall, as measured by the number of reversals in noun objects; and (2) the rated similarity in meaning between the verbs.

Another line of support for a componential model of meaning comes from studies of verification latencies for sentence - picture matching (e.g., True/False "The square is present," given that either a square or a circle is displayed). This line of argument depends on the assumption that certain words contain inherent negative components; for example, it is proposed that the representation of absent is "NOT (representation of pres ent)." In the sentence-picture matching task, a systematic pattern of latencies is found. For affirmative sentences, true sentences are faster than false sentences; but for negative sentences, false sentences are faster than true sentences. The point is that this rather complex pattern of reaction times is found both for explicit negatives, such as the pair present/ isn't present and for implicit negatives, such as present/absent (Carpenter \& Just, 1975; Clark, 1974). This correspondence provides support for the assumption that there is an embedded negative component in absent.

Another psychological phenomenon for which the componential approach has been useful is that of acquisition of meaning. Since Clark's (1973) proposal that children's acquisition of word meaning is best described as the gradual accretion of semantic features, there have been many studies of semantic acquisition based on the componential approach. The semantic features hypothesis ${ }^{1}$ has provided a useful

Feature lists seem a less useful model of semantic representation than more propositional formats, such as networks or propositional formulae. However, many of the empirical and theoretical assertions originally couched in terms of feature lists have natural restatements in terms of propositional forms of representation. 
explanatory framework for acquisition of meaning, predicting both the finding that children acquire the meanings of general words before the meanings of specific words with more semantic components (e.g., give before sell), and the finding that children often initially treat specific words as though they had the same meanings as general words of the same family (e.g., sell understood as give; e.g., Bowerman, Note 1; Clark, 1973; Gentner, 1975, 1978; Kuczaj \& Maratsos, 1975).

The notion of componential representation thus provides a useful explanatory framework for discussing such phenomena as relatedness among word meanings, acquisition of meaning, and the polarity patterns of the chronometric studies. It also provides a useful formalism in which to model semantic integration effects in reading and discourse (Gentner, Note 3 ). These converging phenomena suggest that accessing componential meaning representations is an important aspect of comprehension.

There is, however, an influential body of research that argues against the componential view. This research has tested a set of predictions of the componential model with null results (Kintsch, 1974; Fodor, 1975; Thorndyke, 1975). These predictions are all based on the general intuition that semantically complex concepts are harder to process than semantically simple ones. The implicit assumption governing this work might be termed the Complexity Hypothesis: that the greater the number of semantic components in a word, the more difficult the word will be to process and the greater the memory load it will create (Kintsch, 1974; Fodor, 1975; Thorndyke, 1975). This hypothesis predicts that use of semantically complex words should lead to (1) longer reaction times for processing the words; (2) poorer performance on distracting tasks; and (3) poorer memory for surrounding words than use of semantically simpler words. It is important to note that the Complexity Hypothesis is rarely labeled as such. It tends rather to be implicit; many of its proponents simply assume that complexity effects must follow from componential representation.

Experiments based on the Complexity Hypothesis have failed to provide evidence for semantic decomposition. Kintsch (1974) was one of the first experimenters to investigate componential processing. In a series of experiments designed to test all three of the predictions of the Complexity Hypothesis, he found no systematic differences between his set of semantically complex words and his set of semantically simple words. These results might be suspect, because Kintsch's heavy reliance on the principle of derivational complexity led him in many cases to construct stimuli that differed in syntactic class as well as in semantic complexity (e.g., the pair bake as simple and baker ["one who bakes"] as complex). However, Thorndyke (1975), using sets of verbs classified as semantically simple or complex according to Schank's (1972) conceptual dependency theory, also failed to find evidence that words of greater semantic com- plexity led to longer comprehension times or to poorer memory for the overall sentences (predictions (1) and (3) of the Complexity Hypothesis, respectively) when imagery was controlled for.

This failure to find differences between semantically simple and semantically complex words has led some researchers to conclude that word meanings are processed holistically, not componentially (e.g., Fodor, 1975; Kintsch, 1974). However it is possible that the fault lies not with the notion of componentiality but with the Complexity Hypothesis.

The present study compares the Complexity Hypothesis with an alternative hypothesis: the Connectivity Hypothesis. Whereas the Complexity Hypothesis considers only the number of semantic components in a word's meaning, the Connectivity Hypothesis takes into account the structure of the representation. The Connectivity Hypothesis states that a verb (or other relational term) whose meaning structure sets up more semantic relations between the nouns (or, more precisely, the referentconcepts conveyed by the nouns) in a sentence should lead to greater memory strength between the nouns. Operationally, this means that there should be better cued recall for one noun given the other noun if a highly connective verb is used in a sentence than if a semantically less connective verb is used. This hypothesis is the application to semantic structure of the widely supported general finding that the greater the number of semantic connections between two concepts, the stronger is the memory connection between them (e.g., Bower, 1973; Mandler, 1967).

To compare the Connectivity Hypothesis with the Complexity Hypothesis, let us consider a particular componential model, the LNR model of verb meaning developed at the University of California at San Diego ${ }^{2}$ (Abrahamson, 1975; Gentner, 1975; Rumelhart \& Levin, 1975; Rumelhart \& Norman, 1975). In this model, the meaning structure of the verb specifies the actions, states, changes-of-state, and other semantic relationships that the sentence conveys as holding between the nouns in the sentence. Verb semantic structure is represented as a propositional network specifying the set of interrelated inferences that are normally made when the verb is used. These inferences are written as subpredicates, such as CAUSE or DO. They are represented in relation to one another and to the noun arguments of the sentence. Since most verbs specify several such inferences, these subpredicates act as components of meaning.

The Complexity Hypothesis and the Connectivity Hypothesis make different predictions when applied to these representations. Consider two sentences identical except as to whether the verb is general or specific.

${ }^{2}$ This representational format was developed at the University of California at San Diego, in a seminar headed by David E. Rumelhart and attended by Adele A. Abrahamson, Danielle DuBois, Dedre Gentner, James A. Levin, and Stephen E. Palmer. 
The Complexity Hypothesis states that the additional semantic components in the complex verb will create a greater processing load and require more storage space in memory and thus lead to poorer memory for the overall sentence than will the simple verb (Kintsch, 1974; Thorndyke, 1975). The Connectivity Hypothesis states that the additional semantic components in a verb's meaning will lead to better memory for other parts of the sentences in those cases in which the extra components provide extra semantic connections. Otherwise, the Connectivity Hypothesis predicts no difference between simple and complex verbs in memory effects.

An example will serve to clarify the two positions. Consider the general-specific verb pair give/sold. The sentence shown in Fig. 1,

\section{Ida gave her tenants a clock.}

conveys that Ida did something which caused a change in the state of possession of the clock, such that an initial state in which Ida possessed the clock is replaced by a final state in which her tenants own the clock. ${ }^{3}$ The verb sell is more specific than the verb give. The sentence shown in Fig. 2,

$$
\text { Ida sold her tenants a clock. }
$$

conveys all the information in the give sentence, but provides more information as well: namely, an opposite change of possession of money from Ida's tenants to Ida, as well as some kind of contract or social agreement between Ida and her tenants.

According to the Complexity Hypothesis, this additional semantic information should lead to a greater memory load, thus depressing memory for other items in the same sentence (e.g., Kintsch, 1974; Thorndyke, 1975). This predicts that cued recall for tenants given Ida will be poorer if sell is used than if give is used. The Connectivity Hypothesis makes the opposite prediction. The verb sell conveys additional connective information beyond that conveyed by give between the nouns Ida and tenants. This additional connective information can be seen explicitly in comparison of Figs. 1 and 2: the representation for sell contains more pathways connecting Ida and tenants than the representation for give. Thus, use of sell results in greater memory strength between the nouns than use of give. Therefore, the Connectivity Hypothesis predicts that cued recall for tenants given Ida will be better with sell than with give.

${ }^{3}$ It might be objected that give has a specific meaning "to transfer possession without recompense" as well as the general transfer-of-possession meaning that was assumed here. The general representation used here has both empirical support (Gentner, 1975) and theoretical support (Bendix, 1966; Fillmore, 1966; Schank, 1972). However, in any case, if the specific interpretation had been taken, this would have biased against the predicted results.

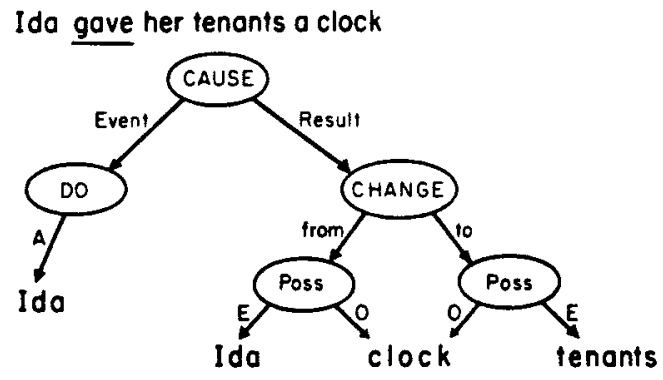

GeNERAL VERB (FEW CONNECTING PATHS)

Fic. 1. Representation of "Ida gave her tenants a clock."

The predictions of the Connectivity Hypothesis arise from the structural properties of the verb representations. In the case of give-sell, the specific verb sell conveys more connective information than the general verb give. Here the Connectivity Hypothesis predicts better object recall for sell than for give, a prediction opposite to that of the Complexity Hypothesis. There are other general-specific verb pairs in which the additional information conveyed by the specific verb does not add further connections between the nouns in the sentence. For these verbs, predictions are different. For example, consider the pair give/mail, as in the sentence shown in Fig. 3,

Ida mailed her tenants a clock.

Ida sold her tenonts o clock

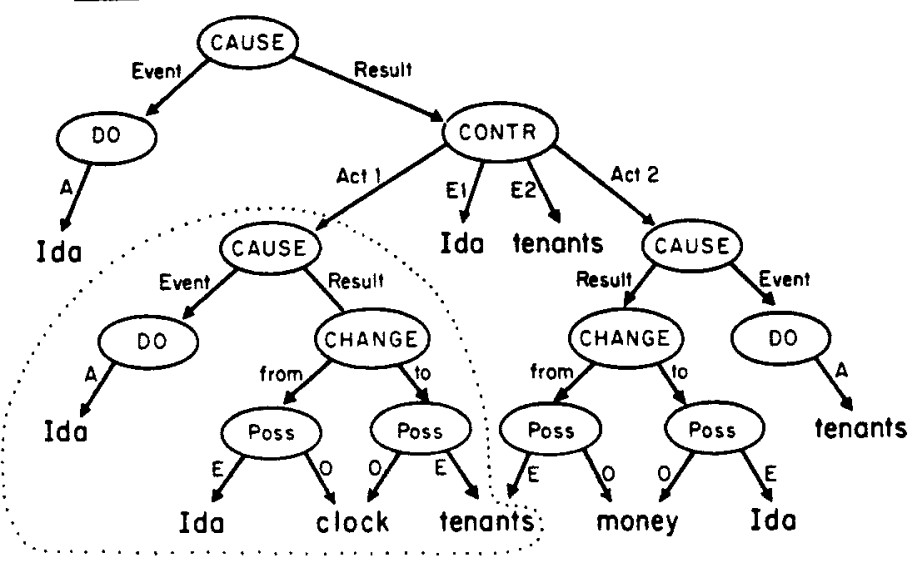

SPECIFIC VERB (MANY CONNECTING PATHS)

FIG. 2. Representation of "Ida sold her tenants a clock." 
Ido maikd her tononis a clock

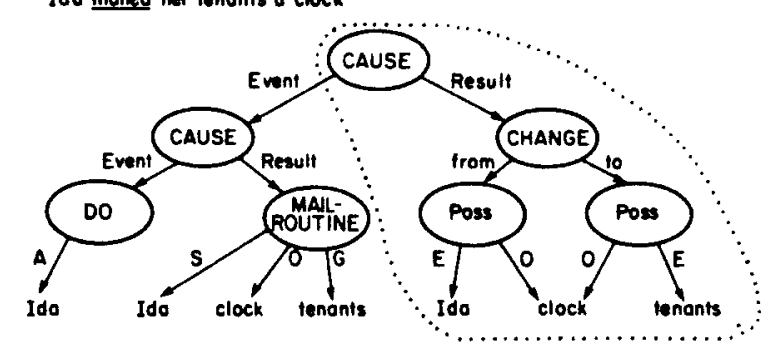

SPECIFIC YERB (FEW CONNECTING PATHS)

FIG. 3. Representation of "Ida mailed her tenants a clock."

The additional subpredicates in mail convey chiefly the actions by which Ida caused the transfer of the clock, and do not, on the whole, serve to connect Ida and tenants more richly than does the general verb give. Thus, for the triad give/mail/sell, the Connectivity Hypothesis and the Complexity Hypothesis predict different patterns of recall of tenants, given Id $a$ as cue. The Connectivity Hypothesis predicts that sell will lead to better memory for tenants than will either mail or give; and further, that mail will lead to roughly the same levels of recall as give. The Complexity Hypothesis predicts that either of the specific verbs-sell or mail- will lead to poorer recall of tenants than will the simpler verb give.

In the experiments reported here, subjects were read sentences containing one of three kinds of verbs: general verbs, connective specific verbs, or nonconnective specific verbs (abbreviated G, CS, and NS, respectively). For each G-CS-NS triad, the CS and NS verbs were more specific members of the same family as the $G$ verb; thus, the meaning representation of the CS and NS verbs included the meaning representation of the $G$ verb as well as additional semantic information. Since both hypotheses depend on structural comparisons among the three representations in each triad, rather than on specific details of each verb's representation, their patterns of predictions would be unaffected by small changes in representational format.

The details of the experiments varied, but in each case the basic measure was the level of recall for the object noun cued by the agent noun, given that a G, CS, or NS verb had been used. For this task, the two decompositional theories yield different patterns of prediction from one another and from any nondecompositional theory. The Connectivity Hypothesis predicts that (1) the level of cued noun recall given CS verbs will be greater than that given $G$ verbs; and (2) the recall levels for NS verbs and $\mathrm{G}$ verbs will be equal. The Complexity Hypothesis predicts that cued noun recall given a $\mathrm{G}$ verb will be better than that given CS or NS verbs.
Notice that complexity effects could occur in two ways: either at input (if complex words require more processing load and thus interfere with processing of surrounding words) or in storage (if complex words require more memory space and so interfere with storage of surrounding words). Thus a failure to find complexity effects would not only tend to disprove the long-term memory predictions of the Complexity Hypothesis but would also place limitations on the processing-load assumptions. The comprehension-time predictions are, of course, not tested here.

Any theory in which the verb is represented as a holistic word-concept rather than as a set of interrelated components predicts no difference in recall among the three kinds of sentences. Further, if a nonverb-central representation, such as Anderson and Bower's (1973) HAM phrasestructure representation, is assumed, then the prediction must be either for no difference among the three kinds of sentences (if a nondecompositional approach is taken) or else that $\mathbf{G}$ sentences will show better object recall than CS and NS sentences (if the Complexity Hypothesis is proposed). Because verbs play no central mediating role between the nouns, it is impossible for HAM to generate the predictions of the Connectivity Hypothesis.

Thus, if no differences are found, we can conclude against both the Complexity Hypothesis and the Connectivity Hypothesis, and we can draw no conclusions concerning verb centrality. If $\mathrm{G}$ sentences are found superior to NS and CS sentences in cued recall of objects, the Connectivity Hypothesis will be disproved and the Complexity Hypothesis supported; and again no conclusions could be drawn concerning verb centrality. If CS sentences are found superior to $G$ and NS sentences, the Complexity Hypothesis will be disproved and the Connectivity Hypothesis supported; the principle of verb-centrality in sentence representation will also be supported.

Experiments 1, 2, and 3 are sentence-memory experiments in which the basic measure was the level of recall of the object nouns given the subject nouns as cues. Experiment 4 is an imagery-rating experiment, included in order to test the possibility that the patterns found in Experiments 1, 2, and 3 were produced by differences in imagery rather than by the hypothesized differences in verb semantic structure.

\section{EXPERIMENT 1}

\section{Subjects}

The subjects were 80 students enrolled in psychology courses at the University of Washington, who received class credit for their participation. Subjects were run in groups of two to six subjects. 
Materials and Design

The stimuli consisted of 16 triads of G/CS/NS sentences, listed in Table 1, and 16 filler sentences. All sentences in a triad had the same agent-subject noun (always a proper name). Each triad had two possible object NP's, chosen to fit with all three of the verb-types used. Thus, a sample triad is

negotiated with (CS)

his housemates.

his neighbors. talked with $(G)$

gossiped with (NS)

Henry Each subject heard only two sentences-the general sentence and one specific sentence-
from each triad. (Although the simplest comparison would have been to present each subject from each triad. (Although the simplest comparison would have been to present each subject
with all three members of each G/CS/NS triad, presenting three such similar sentences might have caused subjects to notice the triads, and possibly to adopt some conscious strategy that

TABLE 1

Sentences Used in Experiment 1

\begin{tabular}{|c|c|c|c|c|}
\hline \multirow[b]{2}{*}{ Name } & \multirow{2}{*}{$\begin{array}{l}\text { General } \\
\text { verb }(G)\end{array}$} & \multicolumn{2}{|c|}{ Specific verbs } & \multirow[b]{2}{*}{ Object noun phrases } \\
\hline & & (NS) & (CS) & \\
\hline Henry & ate & lunched (on) & gnawed (on) & $\begin{array}{l}\text { a pork chop } \\
\text { a tumip }\end{array}$ \\
\hline Henry & cleaned & disinfected & scrubbed & $\begin{array}{l}\text { the bath tub } \\
\text { the bird cage }\end{array}$ \\
\hline Henry & $\begin{array}{l}\text { talked } \\
\text { (with) }\end{array}$ & $\begin{array}{l}\text { gossiped } \\
\text { (with) }\end{array}$ & $\begin{array}{l}\text { negotiated } \\
\text { (with) }\end{array}$ & $\begin{array}{l}\text { his housemates } \\
\text { his neighbor }\end{array}$ \\
\hline Henry & obtained & stole & grabbed & $\begin{array}{l}\text { a bottle of whiskey } \\
\text { the tickets to a rock concert }\end{array}$ \\
\hline Ethyl & altered & shortened & stitched & $\begin{array}{l}\text { a tent } \\
\text { an apron }\end{array}$ \\
\hline Ethy! & greeted & welcomed & hugged & $\begin{array}{l}\text { her grandmother } \\
\text { her chums }\end{array}$ \\
\hline Ethyl & tended & protected & watered & $\begin{array}{l}\text { the orchids } \\
\text { the horses }\end{array}$ \\
\hline Ethyl & damaged & ruined & smashed & $\begin{array}{l}\text { the Tiffany lamp } \\
\text { the hall mirror }\end{array}$ \\
\hline $\operatorname{Max}$ & communicated & confessed & admitted & $\begin{array}{l}\text { his past to his wife } \\
\text { his feelings to the chaplain }\end{array}$ \\
\hline $\operatorname{Max}$ & prepared & chilled & minced & $\begin{array}{l}\text { some clams } \\
\text { some green peppers }\end{array}$ \\
\hline $\operatorname{Max}$ & $\begin{array}{l}\text { went } \\
\text { (across) }\end{array}$ & $\begin{array}{l}\text { hurried } \\
\text { (across) }\end{array}$ & $\begin{array}{l}\text { sprinted } \\
\text { (across) }\end{array}$ & $\begin{array}{l}\text { the intersection } \\
\text { the railroad tracks }\end{array}$ \\
\hline Max & gave & mailed & sold & $\begin{array}{l}\text { his tenants some art posters } \\
\text { his nephew an old clock }\end{array}$ \\
\hline Rebecca & knew & met & married & $\begin{array}{l}\text { a tennis instructor } \\
\text { a musician }\end{array}$ \\
\hline Rebecca & $\begin{array}{l}\text { worked } \\
\text { (on) }\end{array}$ & finished & painted & $\begin{array}{l}\text { some birthday plesents } \\
\text { some party decorations }\end{array}$ \\
\hline Rebecca & $\begin{array}{l}\text { looked } \\
\text { (at) }\end{array}$ & $\begin{array}{l}\text { stared } \\
\text { (at) }\end{array}$ & $\begin{array}{l}\text { glared } \\
\text { (at) }\end{array}$ & $\begin{array}{l}\text { the children in the park } \\
\text { the Veteran's parade }\end{array}$ \\
\hline Rebecca & $\begin{array}{l}\text { took out } \\
\text { (out) }\end{array}$ & unpackaged & $\begin{array}{l}\text { poured } \\
\text { (out) }\end{array}$ & $\begin{array}{l}\text { her dessert } \\
\text { her snack }\end{array}$ \\
\hline
\end{tabular}

would have altered the results.) Subjects were divided into two basic groups, of which one heard $\mathrm{G}$ sentences and NS sentences and the other heard G sentences and CS sentences. Thus a subject in the G/NS group might hear

Henry talked with his neighbors.

Henry gossiped with his housemates.

The two groups of subjects were each further divided into two subgroups in order to counterbalance the pairing of objects and verbs; e.g., a subject in the second G/NS group would hear

\section{Henry talked with his housemates.}

Henry gossiped with his neighbors.

Each subject heard 16 general and 16 specific sentences, as well as 16 filler sentences-a total of 48 sentences. These were presented in two blocks of 24 sentences. Each block featured only two agent-subjects, with four experimental sentence pairs and four filler sentences for each agent. Thus, the first block of sentences might consist of 12 sentences about Ethyl (four general, four specific, and four fillers) and 12 sentences about Henry, presented in semirandom order such that members of a general-specific pair were not presented consecutively. Using the same agent for several different sentences was done in order to foster a naturalistic comprehension situation, rather than presenting subjects with isolated propositions. In line with this aim, the instructions encouraged subjects to form impressions of the protagonists (see Procedure, below).

The design included two between-subjects factors-Pairing of objects and verbs ( 2 levels) and verb Connectivity ( 2 levels: G/NS versus G/CS)-and two within-subjects factorsItems (16 levels) and verb Specificity ( 2 levels: $\mathrm{G}$ versus either $\mathrm{CS}$ or NS).

For both hypotheses, the major predictions concern within-subjects differences. The Complexity Hypothesis predicts, in both the G/NS and the G/CS groups, better object recal with general verbs than with specific verbs, giving a main effect of specificity. The Connectivity Hypothesis predicts better object recall with specific verbs in the G/CS group, and equal object recall for specific and general verbs in the G/NS group. This means there should be a significant interaction between Specificity and Connectivity. Since the $G$ sentences are identical in the G/CS and G/NS conditions, both hypotheses make a secondary betweengroups prediction that the level of object recall will be equal for the $G$ sentences across groups.

\section{Selection of Stimuli}

For each of the general verbs, an NS verb and a CS verb were selected. Both kinds of specific verbs involve additional subpredicates beyond those of the general verb. For the $C S$ verbs, but not for the NS verbs, the additional subpredicates add connections between the two noun arguments selected for testing. In the following discussion I indicate the kinds of amplifications conveyed by each of the NS and CS verbs. Notice that the connectivity of a verb is determined relative to a particular pair of its noun arguments. For example, in the pair give/mail, although mail adds connections between the agent noun and the object transferred, it does not add connections between the agent noun and the recipient (see Fig. 3). Thus mail is an NS verb for the agent-recipient noun pair tested here, though it would have been a CS verb had the agent-object pair been tested. The first noun argument is always the subject noun. The format for verb pairs in this discussion is 
CS Amplifications. In some cases, the CS verb adds to the change-of-state in the G verb a change-of-location (often a further specification of the physical activities by which the agent caused the change of state). An example is grab/obtain (tickets) which tells us that the agent not only caused a change of possession of the tickets (as conveyed by obtain), but that she in doing so caused a change in the location of the tickets (in fact, a rather abrupt change). Other pairs in which the CS verb adds change(s) of location to the meaning of the G verb are gnaw onleat (turnip); stitch/alter (tent); hug/great (chums); water/tend (orchids); smash/damage (lamp); mincelprepare (clams); sprint across/go across (intersection); poured out/took out (snack); paint/work on (presents); and scrub/clean (tub).

In other cases, CS verbs add specification of social transactions between the two noun referents. For example, sell/give (tenants); negotiate with/talk with (neighbor); admit/ communicate (wife); glare at/look at (parade); and marry/know (instructor) all imply social acts or relationships beyond those specified by the general verbs.

NS Amplifications. These amplifications do not further connect the critical pair of nouns. Some NS verbs provide further specification of the final state in a state-change verb; e.g., ruin/damage (lamp). The general verb damage conveys that the agent did something to cause a change in the condition of the lamp such that its final condition was worse than its cause a change in the condition of the lamp such that its final condition was worse than its
initial condition. The NS verb ruin adds the information that the final state was one of total uselessness-but this information does not add any new connections between agent and object. Other cases of final-state specification are shorten/alter (tent); disinfect/clean (tub); chill/prepare (clams); and finish/work on (presents). One case of initial-state specification was included: unpackaged/took out (snack).

Another kind of NS amplification is information about overall timing; thus, lunch onleat (turnip) specifies the time of the ingestion, but does not add further connections between agent and object; hurry across/go across (intersection) specifies a speedy completion of the change-of-location; meet/know (instructor) specifies that the acquaintance is just beginning; and stare at/look at (parade) specifies that the looking went on for some time (and perhaps also indicates the intensity of the looking).

In some cases, the NS verb provides a fuller description of a noun argument other than one of the critical pair. This occurs with confess/communicate (wife) and gossip/talk with (neighbor); the NS verb specifies the content of the discussion, but does not add connections between the two discussants.

Other instances of nonconnecting amplifications are protect/tend (orchids), which conveys the existence of an outside entity against which the agent must contend in order to maintain the orchids; steallobtain (tickets), which conveys that the change of possession was illegal with respect to the previous owner; welcometgreet (chums), which adds that the greeting is taking place on the agent's territory; and mail/give (tenants), which specifies the actions performed by agent on object, but does not add to the relation between agent and recipient.

It should be noted that these representations are tentative, and undoubtedly some aspects could be improved. However, if the general framework is correct, then we should find the predicted differences among the three verb categories.

\section{Procedure}

Subjects were told that they would hear sentences about two people and that they had two tasks: first, to use the sentences to form an impression of each of the people; and second, to remember the sentences as accurately as possibie. They were told that they would be asked to write a short impression of each character, focusing on any aspect of the person that had struck them, and also that they would be tested on their memory for the sentences.

In each of the two blocks of sentences, the first two sentences presented were filler sentences that physically described the characters: e.g., "Max is dark-haired and handsome." After this, the six remaining filler sentences and 16 experimental sentences were read to the subjects once each, in semirandom order, with a 3- to 4-sec pause between sentences. After each block of sentences, the subjects were given a sheet with the names of the two agent-nouns and told to write, first, a very brief description of each person; and second, all the sentences they could remember about each person, as accurately as possible. After about $1 \mathrm{~min}$, subjects who had not done so were urged to complete their descriptions and begin recall. Recall was self-paced, and took about $8 \mathrm{~min}$. After all subjects had finished recalling sentences, the sheets were collected and the next block of sentences was read.

\section{Scoring}

The sentences were scored as to correct (verbatim) recall of the object nouns. (A lenient scoring method, in which object synonyms were also accepted, yielded the same pattern of results.) Scoring of objects was independent of whether the verb of the sentence was accurately recalled. This was done to allow for the fact that verbatim memory for verbs is generally poor (e.g., Reynolds \& Flagg, 1976). To avoid having to compare the similarity of verb intrusions, which might have led to some subtle bias, object nouns were scored regardless of the accuracy of their verbs.

\section{Results and Discussion}

The results of the agent-cued recall, shown in Fig. 4, provide support for the major, within-subjects predictions of the Connectivity Hypothesis and tend to disconfirm the Complexity Hypothesis.

As predicted, subjects in the G/CS condition showed better recall of objects for the specific sentences than for the general sentences. Subjects in the $\mathrm{G} / \mathrm{NS}$ condition showed no difference between general and specific sentences in the number of objects recalled. In terms of the design used here, this effect is tested by examining the interaction between the between-subjects factor of Connectivity and the within-subjects factor of Specificity; this interaction is significant $(F(1,76)=7.0, p<.01)$.

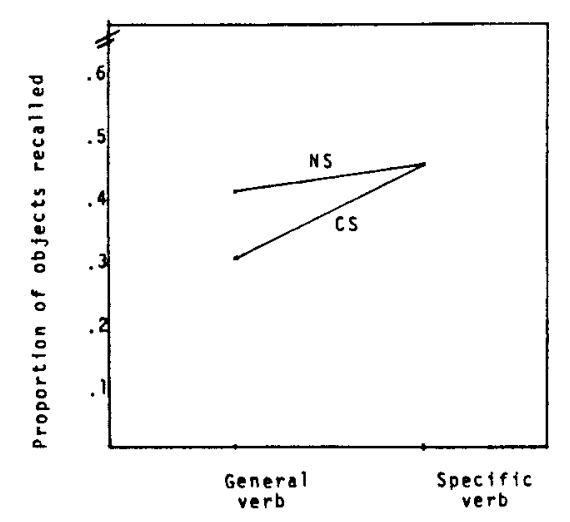

Fig. 4. Results of Experiment 1: Proportion of object nouns recalled given subject nouns as cues. 
In addition to the significant $\mathrm{C} \times \mathrm{S}$ interaction predicted, both Connectivity and Specificity were significant as main effects $(F(1,76)=5.3, p<$ .05 and $F(1,76)=20.3, p<.01$, respectively). The other significant effects were Items $(F(15,1140)=4.3, p<.01)$; Pairing X Items $(F(15,1140)=2.5$, $p<.01)$; Connectivity $\times$ Pairing $\times$ Items $(F(15,1140)=2.0, p<.01)$; and Pairing $\times$ Items $\times$ Specificity $(F(15,1140)=3.8, p<.01)$. The significance of the factor of Items (referring to the 16 triads) and of the various interactions involving Items and Pairings (i.e., within each sentence, the two possible pairings of verbs with objects) indicates that there were differences within the stimulus materials. Therefore, an analysis over items was performed.

Item analysis. The difference between $\mathrm{G}, \mathrm{CS}$, and NS conditions (with the $G$ scores taken as the average of the separate $G$ scores obtained for the $\mathrm{G} / \mathrm{CS}$ groups and the G/NS groups) was significant when analyzed over items $(F(2,40)=5.27, p<.01)$. In addition, individual $t$ tests for general-specific differences, analyzed across items as planned comparisons were carried out. Separate comparisons were made for the G/CS and $\mathrm{G} / \mathrm{NS}$ groups. In the $\mathrm{G} / \mathrm{CS}$ group, the level of object recall was significantly higher for $\mathrm{CS}$ verbs than for $\mathrm{G}$ verbs $(t(15)=5.30, p<.0005$, one-tailed). The $\mathrm{G}-\mathrm{NS}$ difference was nonsignificant $(t(15)=1.15)$. The superiority of CS over $\mathrm{G}$ sentences is predicted by both hypotheses; but the lack of difference between $G$ and NS sentences accords only with the Connectivity Hypothesis.

While the results. found in Experiment 1 conformed to the withinsubject predictions of the Connectivity Hypothesis, there were some discrepancies in the secondary, between-subjects findings. The prediction was that the two groups of subjects would have identical levels of noun recall on the $G$ sentences. The G/CS group, but not the G/NS group, was predicted to show higher recall in the specific-verb sentences. Thus, the overall performance level of the $\mathrm{G} / \mathrm{CS}$ group should have been higher than that of the G/NS group. Instead, the G/CS group performed worse overall than the G/NS group. The two groups showed equal recall levels in the specific-verb sentences, with the G/CS group performing worse than the G/NS group on the G sentences. Thus, although the within-group differences are as predicted, the between-group differences are not. These between-group differences could mean that something important was missing from the theory, or they might have arisen from simple group differences.

In order to differentiate between these two explanations, Experiment 2 was performed. A second reason for this experiment was that the memory load of 24 sentences per block in Experiment 1 seemed rather high. Experiment 2 was a replication of Experiment 1, with the number of stimuli per block lowered to reduce the possible overloading problem. If the intergroup differences had persisted, a revision of the theory would have been required.

\section{EXPERIMENT 2}

Experiment 2 was similar in materials and design to Experiment 1 , except that four blocks of sentences were used in Experiment 2, instead of two blocks. This allowed fewer sentences per block.

\section{Subjects}

Subjects were 16 undergraduate students at the University of Washington, who received class credit for their participation.

\section{Materials and Design}

Each block contained eight experimental sentences and six filler sentences. As in Experiment 1 , each block featured two characters, each of whom figured as agent in one-half of the sentences. The experimental stimuli were, with only minor changes, those used in Experiment 1: i.e., 16 triads of sentences, each sentence composed of an agent noun (a proper name), a verb (G, CS, or NS), and one of two object NP's. The design was as in Experiment 1.

\section{Procedure}

The procedure was as in Experiment 1, except that there were four blocks of 14 sentences instead of two blocks of 24 sentences. As in Experiment 1, after each block subjects were given a sheet containing the names of the two characters featured in that block and told to write a brief impression of each, followed by all the sentences they could remember about each of the two characters. Verbatim recall of objects was scored.

\section{Results}

The major within-subjects results, shown in Fig. 5, are as predicted by the Connectivity Hypothesis. Subjects in the G/CS condition recalled

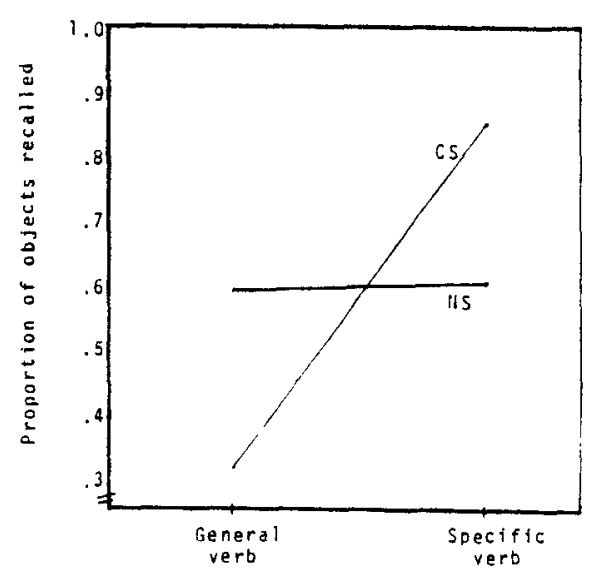

Fig. 5. Results of Experiment 2: Proportion of object nouns recalled given subject nouns as cues. 
many more objects for specific verbs than for general verbs. Subjects in the G/NS condition did not show such a difference, providing support for the Connectivity Hypothesis over the Complexity Hypothesis. This pattern is reflected in the significant interaction of Connectivity $\times$ Specificity $(F(1,12)=33.4, p<.01)$.

The main effect of Specificity is also significant $(F(1,12)=33.9, p<$ $.01)$. Other significant effects are the main effect of Items $(F(15,180)=$ $2.8 ; p<.01)$ and the following interactions: Connectivity $\times$ Items $(F(15,180)=2.3, p<.01)$; and Pairing $\times \operatorname{Items}(F(15,180)=1.8, p<.05)$.

Item analysis. This difference between G, NS, and CS conditions (with the $\mathrm{G}$ scores taken as the average of the separate $\mathrm{G}$ scores obtained for the $\mathrm{G} / \mathrm{NS}$ subjects and the G/CS subjects) was also significant when analyzed over items $(F(2,45)=18.2, p<.01)$. Separate $t$ tests for differences were computed as planned comparisons within the $\mathrm{G} / \mathrm{CS}$ and $\mathrm{G} / \mathrm{NS}$ conditions. As predicted, the G/CS difference was significant $(t(15)=13.73, p<$ .001 ), while the $\mathrm{G} / \mathrm{NS}$ difference was nonsignificant $(t(15)=.362)$.

The within-subjects results of Experiment 2 again tend to disconfirm the Complexity Hypothesis and support the Connectivity Hypothesis. In addition, these results are more consistent with the secondary, betweengroups predictions made by both hypotheses than were the results of Experiment 1. In particular, the G/CS group is equal in overall performance to the G/NS group, suggesting that the inferiority found in Experiment 1 was a chance effect. However, there is still one between-groups discrepancy that requires explanation: here, as in Experiment 1, recall of the objects of $G$ verbs is lower in the G/CS condition than in the G/NS condition. This is disturbing, since neither hypothesis predicts a between-group difference in the behavior of the general verbs. Although the G/CS group showed superior performance on the specific sentences, their performance on the $G$ sentences was lower than that of the G/NS subjects. This effect also occurred in Experiment 1. There seems to be a systematic drop in $\mathrm{G}$ performances for the G/CS group as compared to the G/NS group.

The explanation for this drop appears to lie in an interference phenomenon whereby recall of an item in a free recall list inhibits the subsequent retrieval of other items from the same list (Rundus, 1973; Slamecka 1968). If, after learning a list of words, a subject recalls or is given a subset of those words prior to free recall of the remaining items, recall of the remaining items is lower. Rundus argued that this recall interference arises when the items share the same retrieval cues, since early-recalled items will block recall of other items associated with the same cue. This argument applies to the results of Experiments 1 and 2. In both experiments, the general and specific sentences of a triad had the same agentnoun retrieval cue. If, as predicted by the Connectivity Hypothesis, CS sentences are more recallable than NS sentences, then the G/CS group should recall a disproportionate number of specific sentences. By the interference effect, this will result in the G/CS group's recalling fewer $G$ sentences for the same agents than the G/NS group.

In these experiments, the verbs in a triad (and in fact in several different triads) shared the same agent nouns. This design was chosen in order to foster a naturalistic situation in which the sentences would function as rich descriptions of the agent-protagonists rather than as lists of isolated propositions. However, it seemed likely that this sharing of agents led to interference effects. Although these effects in no way contradict the Connectivity Hypothesis (and indeed depend upon the superior recallability of CS sentences for their existence), it seemed desirable to sort out the phenomena more clearly. Therefore, a third experiment was conducted. The design was altered to minimize interference effects: no two sentences shared the same agent-noun, and only one verb from each triad was presented to a given subject. Under these conditions, the Connectivity Hypothesis predicts that CS performance will be superior to $G$ and NS performance, which will be equal. The Complexity Hypothesis predicts that performance on $\mathrm{G}$ sentences will be superior to performance on NS and CS sentences.

\section{EXPERIMENT 3}

In Experiments 1 and 2, half the subjects heard G/CS pairs and half heard G/NS pairs. This design allowed a within-subject comparison of two of the verbs from the same triad. However, only one comparison-G/CS or G/NS-could be made within any subject. In Experiment 3, the pattern was changed so that each subject received one-third $G$, one-third $C S$, and one-third NS sentences, each from different triads. The G/CS/NS comparison was thus within-subjects but not within-triads, for a given subject. Thus, one group of subjects would hear

Douglas talked with his neighbor. (G)

Henry stitched a tent. (CS)

Cynthia finished the chest of drawers. (NS)

The second group would hear

Douglas gossiped with his neighbor. (NS)

Henry altered a tent. (G)

Cynthia painted the chest of drawers. (CS)

The third group would hear

Douglas argued with his neighbor. (CS)

Henry shortened a tent. (NS)

Cynthia worked on the chest of drawers. (G) 
There were other differences in materials between Experiments 1 and 2 and Experiment 3. First, in Experiment 3, each character appeared in only one sentence, instead of appearing in several sentences as in Experiments 1 and 2. Second, for Experiment 3 two new triads were added to make 18 triads instead of 16 as in Experiments 1 and 2. Finally, some changes in the stimuli were made. Prior to constructing the stimuli for Experiment 3, ratings of verb-object appropriateness using 10 naive undergraduate students as judges were obtained for all the verb-object combinations used in Experiments 1 and 2, as well as other possible combinations. These were used to select for each triad an object noun which was rated as equally appropriate for each of the three verbs in the triad.

\section{Subjects}

The subjects were 18 students enrolled in psychology courses at the University of Washington, who received class credit for their participation. Subjects were divided into three stimulus-groups, as described below, and were run in groups of two to six subjects.

\section{Stimuli}

The stimuli, listed in Table 2, consisted of 18 triads of subject-verb-object sentences and six subject-verb-object filler sentences. Of the 18 triads, 16 utilized the same verbs as were used in Experiments 1 and 2, with minor changes in the object nouns. The other two triads were new. All sentences in a triad had the same agent-subject, a proper name, and the same object NP; they differed only in the verb (G, NS, or CS). Each subject heard only one member of each triad. There were three groups of subjects; each group heard a differen one-third G, one-third CS, and one-third NS sentences. The six filler sentences, also listed in Table 2, had different agents, verbs, and objects from the experimental sentences. Thus, each subject heard a total of 24 sentences, each with a different agent, verb, and object. The sentences were presented in three blocks of six experimental and two filler sentences.

\section{Procedure}

Subjects were told that they would hear sentences about people and that they had two tasks: to understand each sentence meaningfully, as though in real life, and to remember the sentences as accurately as possible. They were not told, as in Experiments 1 and 2, to form personality impressions of the characters. In each of the three blocks, one filler sentence preceded and one followed the six experimental sentences. The method of presentation and testing was the same as for Experiments 1 and 2, except that subjects did not write out impressions of the characters, but instead were given sheets containing the names of the eight agent nouns (including the names used in the fillers) and asked to begin directly on the recall task. Verbatim recall of objects was scored, as in Experiments 1 and 2.

\section{Results}

The results, shown in Fig. 6, conformed to the predictions of the Connectivity Hypothesis. More objects were recalled for CS sentences than for sentences containing either $\mathrm{G}$ verbs or NS verbs $(F(2,30)=3.4, p<$ $.05)$. No other effects were significant in the $3 \times 3 \times 3$ analysis of variance (Subject Group $\times$ Block $\times$ Verb Type).

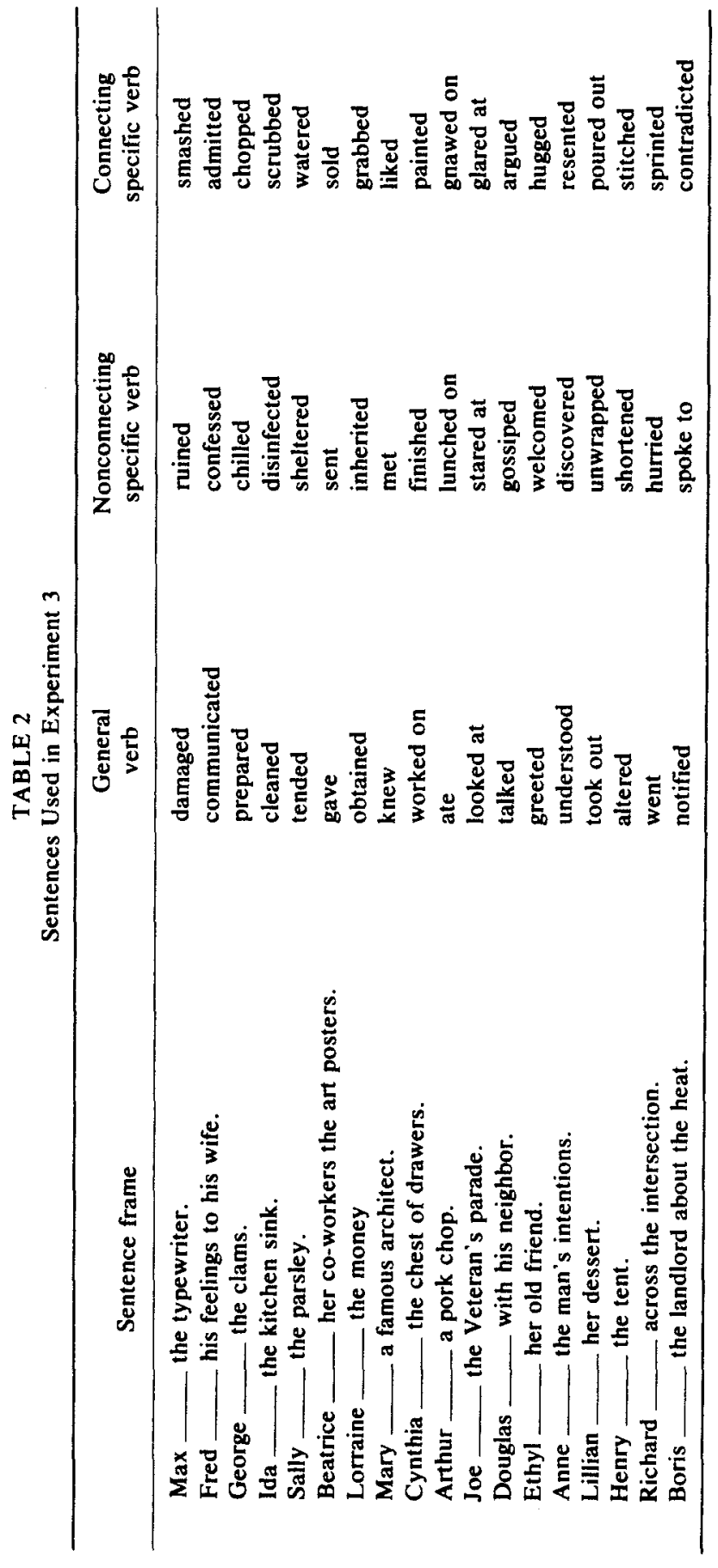




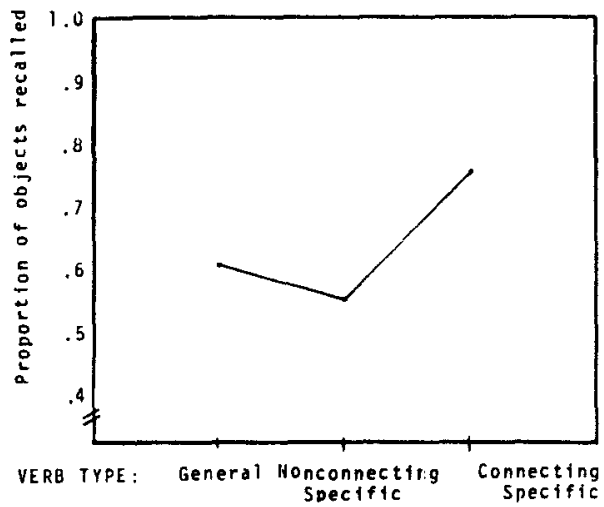

FiG. 6. Results of Experiment 3: Proportion of object nouns recalled given subject nouns as cues.

Item analysis. The difference between the G, CS, and NS conditions was also significant when analyzed over items $(F(2,51)=3.94, p<.05)$. As planned comparisons, $t$ tests for differences were computed across items for the three pairs of means. As predicted, the level of object recall in the CS condition was significantly different from that in the $\mathrm{G}$ condition $(t(17)=3.19, p<.005)$ and from that in the NS condition $(t(17)=2.85, p$ $<.01)$. The NS and G conditions did not differ significantly from one another $(t(17)=.37)$.

Thus the major predictions of the Connectivity Hypothesis are again confirmed. The similar performance of $G$ and NS verbs under this design is consistent with the claim that the lowered performance on $G$ sentences in the $\mathrm{G} / \mathrm{CS}$ condition in Experiments 1 and 2 was due to retrieval interference effects. Since these interference effects themselves depend upon CS-superiority for their existence, the overall pattern of results is strong support for the Connectivity Hypothesis over the Complexity $\mathrm{Hy}$ pothesis.

\section{EXPERIMENT 4}

One possibility that might have required a reinterpretation of the results was that the CS verbs lead to better object recall simply because they produce greater imagery than the NS verbs or the $G$ verbs. The theoretical status of imagery as a causal explanation is not at all clear. Nevertheless, use of high-imagery connectors has been shown to improve sentence memory (e.g., Paivio, 1971; Thorndyke, 1975), so it is desirable to show that the present results cannot be accounted for by imagery instead of connectivity. Therefore, imagery ratings of the materials used in these experiments were obtained and correlated with the recall levels in Experiments 1,2 , and 3 .
A priori, we might expect a weak correlation between rated imagery and connectivity. High-imagery verbs can be characterized as conveying more visual-spatial information than low-imagery verbs. To the extent that this information concerns spatial relations between the noun arguments, highly imageable verbs will be more connective than their general counterparts (e.g., smash vs damage). However, not all imagery is connective; and moreover, there are many nonspatial relationships that can enter into word meaning and that can serve a connective function. Therefore a high correlation would place the Connectivity Hypothesis as stated in question.

\section{Subjects}

Subjects were 48 college students living in the Boston-Cambridge area, who were recruited by newspaper advertisements and paid for their participation.

\section{Materials and Design}

Each subject rated only one member of each triad, to avoid the possibility of carryover between same-triad sentences. The subjects were divided into four groups of 12 persons each. Three of the groups each rated one of the three sets of sentences used in Experiment 3. These sentence-sets, each containing 18 sentences, were constructed so that only one sentence from each triad appeared, and equal numbers of G, CS, and NS sentences appeared. The fourth group of subjects rated a set of eight odd sentences that had appeared in Experiments 1 and 2, but not Experiment 3.

\section{Procedure}

Subjects were given rating sheets ranging from 1 (extremely low imagery) to 5 (extremely high imagery) and asked to rate each sentence for its imagery value. Each group of subjects heard a different random order of sentences. Four filler sentences preceded and two followed the list.

\section{Results}

The mean ratings ranged from 1.92 to 4.92 . The mean ratings by condition were 3.59 for $G$ verbs, 3.45 for NS verbs, and 3.83 for CS verbs. Imagery was uncorrelated with the recall level on any of the three experiments. For each of the experiments, a Spearman rank order correlation was performed between the mean imagery rating and the level of object recall obtained for each sentence. All correlations were nonsignificant. For Experiment 1, $r=-.10 ; z=-.71$. For Experiment $2, r=$ $.04 ; z=.25$. For Experiment $3, r=-.08 ; z=-.56$

Every combination of imagery level and recall level was found. There were verbs that were high in both imagery and recall level (e.g., the CS verbs smash and $h u g$ ) and verbs that were low in both (e.g., the G verb communicate and the NS verb finish). There were also high-imagery lowrecall verbs such as lunch on (NS); and clean (G); and low-imagery highrecall verbs such as marry (CS) and admit (CS). The lack of imagery 
correlation in Experiment 4 undermines rather seriously any attempt to explain the recall differences here in terms of imagery.

\section{Word Frequency}

For the sake of completeness, rank-order correlations were also performed between level of object recall and word frequency of the verb (Kučera \& Francis, 1967). For Experiment 1, $r=-3.2 ; z=-2.9 ; p<.05$. For Experiment 2, $r=-.12 ; z=.84$, nonsignificant. For Experiment 3, $r=.11 ; z=.77$, nonsignificant. The one significant correlation was negative, and may reflect the fact that CS verbs were usually lower in frequency than $G$ verbs. This difference in frequency meant that the $C S$ sentence of a triad had a lower combined word-frequency than the $G$ sentences. This should, if anything, have biased against the predicted CS superiority, since it meant that noun recall was mediated by lowerfrequency items in CS sentences than in $G$ sentences.

\section{DISCUSSION}

In these cued-recall experiments, object nouns were better recalled when connective specific verbs were used than when general verbs or nonconnective specific verbs were used. These systematic effects of verb semantic structures on memory are evidence for componential representation of meaning. Further, this pattern of findings is evidence for the Connectivity Hypothesis. This hypothesis states that verbs whose semantic structures provide many semantic connections between the nouns in a sentence will lead to greater memory strength between those nouns than verbs whose underlying structures contain few connections. The Connectivity Hypothesis derives from two principles specific to representation of sentence meaning, and one general processing principle. The two specific representational principles are (1) that verbs provide central relational frames for sentences; and (2) that the verb is stored in long-term sentence memory as a componential network of subpredicates. The general processing principle is the principle of connectivity: that ability to remember one concept given another increases with the number of stored semantic connections between the two concepts. This basic principle has been supported in previous memory research (e.g., Bower, 1973; Mandler, 1967); only the application to decompositional semantic structures is new.

Previous work on the psychological reality of a semantic substructure for word meaning has been based on the Complexity Hypothesis. Negative results have been taken as evidence not against the complexity assumptions, but against the notion of componential representation. However, if we shift the focus of questioning to the Complexity Hypothesis itself, then the work of Kintsch (1974) and Thorndyke (1975) accords with the present research in disconfirming the Complexity Hypothesis as applied to memory. There is no evidence that use of semantically complex words depresses memory for the other words in a sentence. Indeed, the connectivity effect found in the present study provides a particularly strong counterexample. The CS verbs, though semantically more complex than the $G$ verbs, lead to better memory for other words in the sentence than do the $G$ verbs; this is the direct opposite of the pattern predicted by the Complexity Hypothesis. The failure to find any difference between $G$ verbs and NS verbs is again an indication of the lack of any effect on memory of complexity per se.

These two hypotheses have different motivating assumptions. The Complexity Hypothesis is motivated by the "bin" view of memory, in which the capacity limitations of various stages of memorial processing form a central theoretical notion. The Connectivity Hypothesis is based on a more structural view of memory, in which the representational assumptions are crucial. The predictions of the Complexity Hypothesis derive from considering long-term memory as a limited-capacity storage system. The predictions of the Connectivity Hypothesis derive from considering memory as a structured set of concepts and relationships, in which greater connectivity between two concepts leads to higher probability of retrieving one concept given the other. Thus the number of semantic components in a representation is an important predictor in the Complexity Hypothesis, but not necessarily in the Connectivity Hypothesis; while the structure of the representations is crucial in the Connectivity Hypothesis, but not in the Complexity Hypothesis. The present results provide support for a more structural view of memory.

Although the focus of this research is on long-term memory predictions, complexity effects could also have occurred during comprehension. The more complex verbs could have created greater processing loads, thus depressing memory for their nouns by competing for working memory resources. Though no such effects occurred here, there may well be complexity effects on processing load in some cases, perhaps those in which the material is less meaningful and familiar than the words used here. (However, from the fact that the only significant correlation between recall level and frequency was negative, we can infer that obtaining a connectivity effect instead of a complexity effect does not depend on use of extremely high-frequency items.) The studies of Kintsch and Thorndyke also failed to provide evidence for the input predictions of the Complexity Hypothesis: that the time and attention required to comprehend a word increases with the number of semantic components in the word. Indeed, so far, the evidence for an effect of semantic composition on comprehension time suggests that something more than mere number of components is operative. In the sentence-picture matching task in- 
volving pairs of positive-negative terms, analysis of the patterns of latencies supported a model in which words containing an extra negative component (e.g., absent) take longer to encode than their positive counterparts (e.g., present) which lack the negative component (Clark, 1974; Carpenter \& Just, 1975; Clark \& Chase, 1972). The latencies were predicted by means of a detailed model featuring separate time parameters for each kind of semantic component. That different components required different time parameters suggests that the time differences between positive and negative terms can be more plausibly explained in terms of structural considerations-such as the role of the negative component in the verification process-than by the merely numerical difference of the one additional negative component.

The failure to find complexity effects in memory does not invalidate other complexity predictions. Although there is at present no evidence for the Complexity Hypothesis in latency tasks, the role of componential structure in time-to-comprehend needs further investigation. There is considerable evidence for complexity effects in acquisition of word meaning (Clark, 1973; Gentner, 1975). This suggests that complexity effects may be most likely to occur with unfamiliar materials. But it is possible that these effects can be detected by more sensitive tests, even with materials that are well-learned. In any case, however strong our intuition that complexity must have a role in adult processing, there seems every reason to disengage tests of the Complexity Hypothesis from tests of the notion of componential storage.

\section{Models of Sentence Representation}

The results of these studies bear on the issues of verb centrality and semantic decomposition, and therefore can help in evaluation of different models. In particular, the Kintsch model, the HAM model of Anderson and Bower, and the LNR model make contrasting predictions. The Kintsch (1974) model is verb-centered and noncomponential. A casegrammar-like representation is assumed, in which the verb is the central relational concept connecting the nouns in the sentence. Words do not decompose during comprehension, but instead are mapped onto wholeword concepts in memory. Meaning rules can then be applied to yield inferences appropriate to the task and the context, but the basic form of long-term storage is in terms of whole-word concepts. The HAM model is nonverb-central and does not utilize decomposition (Anderson \& Bower, 1973). HAM assumes a binary phrase structure for long-term sentence representation, in which a sentence is divided into subject and predicate, with the verb appearing as one of the elements in the predicate. Thus semantic connections between the nouns in a sentence are not mediated by the verb. On the issue of decomposition, Anderson and Bower (1973) have remarked that a componential treatment might be useful, but the HAM model does not utilize representations below the word level, and experimental predictions based on the model have generally assumed memory for the precise words used in the input string. In the LNR verb schema model used here, it is not words or word-concepts, but rather their componential representations that are stored in memory (Gentner, 1975; Munro, 1975; Rumelhart \& Levin, 1975). Further, the representation of the verb occupies a central connective role in memory for sentences. Thus, changes in verb semantic structure should affect memory for the sentence as a whole. The Conceptual Dependency model of Schank and his co-workers posits a decompositional and basically verbcentral structure for sentence meaning (Schank, 1972; Schank \& Colby, 1973; Schank et al., Note 5). This model would make the same predictions as the LNR model, given similar processing assumptions.

Overall, the four models differ in two ways: (1) The LNR model, the Conceptual Dependency model and the Kintsch model posit verbcentered structure, while the HAM model posits a nonverb-central binary phrase structure; (2) Decomposition during comprehension is an explicit and important assumption in the LNR model and in the Conceptual Dependency model; the HAM model is in practice nondecompositional; and the Kintsch model explicitly assumes no decomposition during comprehension. The finding that changes in verb meaning structure affect memory strength between nouns disconfirms both the HAM model and Kintsch's model, for different reasons. HAM is ruled out by the verbcentrality evidence that verbs' semantic structures mediate between nouns in memory. Kintsch's model is ruled out by the evidence in favor of componential storage. A verb-central componential model is supported, such as the LNR model or the Conceptual Dependency model.

Decomposing "Decomposition"

The semantic decomposition position has come to include a rather complex combination of representational and processing assumptions. Four tacit, but nevertheless strong, assumptions that tend to be associated with the semantic decomposition position are (1) that a word's meaning representation must satisfy necessary and sufficient conditions for use; (2) that the componential representation is exhaustive of the word's meaning; (3) that the entire content of a word's representation is accessed in a context-independent fashion during comprehension; and (4) that the components are nondecomposable elements of a primitive base. (Assumptions (1) and (2) are closely related.) A fifth assumption, that a word meaning is invariant (within any given word-sense) follows largely from assumptions (2) and (3), which taken together imply that the 
exhaustive meaning representation for any given word must be accessed without considering context.

The opposing position, that words are not decomposed during comprehension, is most often associated with some form of the meaningpostulate position: that the immediate result of sentence comprehension is simply to access a meaning-concept for each of the words in the sentence, and that inferencing is done later by a set of logical rules or relationships called meaning-postulates (Fodor, 1975; Kintsch, 1974; see also Carnap, 1947, pp. 122-129). Thus, in Fodor's example pair of bachelor/unmarried man, he argues that it is not the case that bachelor is represented in terms of components for "unmarried" and "man." Rather, bachelor and unmarried man have independent whole-word concept representations in the lexicon. Accessing these representations constitutes sentenceunderstanding. Rules of inference, including those that express the semantic connections between the two concepts, can be applied after this sentence-understanding stage, if warranted. The typical meaningpostulate position differs from the typical decompositional position in (1) rejecting a necessary-and-sufficient definitional format for meaning; (2) rejecting the notion of exhaustive meaning representations; (3) placing inferencing after comprehension in time, not during comprehension; and (4) assuming that word meanings are stored as holistic concepts. This model has the advantage of allowing great contextual variability, since the choice of which inferences to make can be postponed until sufficient contextual information has been collected. However, this advantage may be illusory, since the context itself is composed of words which must be interpreted before they can play the role of contextual background for other words.

The point is that both the typical decompositional position and the typical meaning-postulate position are conflations of separable assumptions. In particular, the content issue of whether meaning representations should be exhaustive and should express necessary-and-sufficient conditions for use can be separated from the processing issue of the timecourse of accessing the representations, and from the structural issue of the degree of componentiality of the representations.

The model proposed here, which might be called the Central Components model, differs from both the extreme decompositional model and the extreme meaning-postulate model. In the Central Components model, the verb's representation is intended to specify the pattern of inferences that is most dependably activated when the verb is comprehended. This model is clearly decompositional; it assumes that one verb leads to several separable (though structurally related) inferences, and that both lexical generalities and psychological phenomena can be stated in terms of connected sets of subpredicates embodying these inferences. However, these inferences are not intended to embody necessary-and-sufficient conditions for use. Instead, the representation offered here for a given verb expresses the central set of inferences (the set most frequently and reliably associated with the verb's use). The representations are not put forth as exhaustive; indeed it is very clear that they are not. For example, the verb give clearly has other possible inferences beyond those specified in Fig. 1: that the giver is generous, that she has the means to give away objects, and so on. There is no fixed stopping point for this kind of inferential processing. Further, the subpredicates are not required to be atoms belonging to a primitive base. A component is useful in a psychological representation if it functions as a familiar unit at that level of representation. Components at one level of representation may be decomposed at the next level down into a further network of linked components.

The present results do not address the time-course of decomposition. However, they are compatible with the position that inferencing begins on-line, before the syntactic parse is completed (Tyler \& Marslen-Wilson, 1977) rather than after the sentence-understanding stage, as Fodor (1975) has proposed. Inferencing would begin with the central set and continue, radiating outward into more esoteric inferences, if the central set of inferences is not sufficient for a satisfactory interpretation of the sentence (cf., Collins \& Loftus, 1975). In summary, the Central Components model is componential in assuming that a structural set of subpredicates is conveyed by the verb. But it does not assume necessary-and-sufficient conditions nor exhaustiveness in representation. Finally, it seems compatible with the assumption that the process of accessing word meanings begins on-line, during comprehension.

The studies described here provide evidence for the psychological reality of semantic structure in representation of sentence meaning, and for a verb-central model of sentence storage. These results further disconfirm the Complexity Hypothesis and provide support for the Connectivity $\mathrm{Hy}$ pothesis.

\section{REFERENCES}

Abrahamson, A. A. Experimental analysis of the semantics of movement. In D. A. Norman \& D. E. Rumelhart (Eds.), Explorations in cognition. San Francisco: Freeman, 1975. Anderson, J. R., \& Bower, G. Human associative memory. Washington, D.C.: Winston \& Sons, 1973.

Bendix, E. H. Componential analysis of general vocabulary: The semantic structure of a set of verbs in English, Hindi and Japanese. The Hague: Mouton, 1966.

Bendix, E. H. The data of semantic description. In D. D. Steinberg \& L. A. Jakobovits (Eds.), Semantics. New York: Cambridge University Press, 1971. 
Bierwisch, M. On classifying semantic features. In D. D. Steinberg \& L. A. Jakobovits (Eds.), Semantics. New York: Cambridge University Press, 1971.

Bower, G. H. Mental imagery and associative learning. In L. Gregg (Ed.), Cognition in learning and memory. New York: Wiley, 1973.

Carnap, R. Meaning and necessity. Chicago: The University of Chicago Press, 1947.

Carpenter, P. A., \& Just, M. A. Sentence comprehension: A psycholinguistic processing model of verification. Psychological Review, 1975, 82, 45-73.

Chafe, W. L. Discourse structure and human knowledge. In J. B. Carroll \& R. O. Freedle (Eds.), Language comprehension and the acquisition of knowledge. Washington, D.C.: V. H. Winston, 1970.

Clark, E. V. What's in a word: On the child's acquisition of semantics in his first language. In T. E. Moore (Ed.), Cognitive development and the acquisition of language. New York: Academic Press, 1973.

Clark, H. H. Semantics and comprehension. In T. A. Sebeok (Ed.), Current trends in linguistics: Volume 12: Linguistics and adjacent arts and sciences. The Hague: Mouton, 1974.

Clark, H. H., \& Chase, W. G. On the process of comparing sentences against pictures. Cognitive Psychology, 1972, 3, 472-517.

Collins, A. M., \& Loftus, E. F. A spreading activation theory of semantic processing. Psychological Review, 1975, 82, 407-428.

Fillenbaum, S., \& Rapoport, A. Verbs of judging, judged: A case study. Journal of Verbal Learning and Verbal Behavior, 1974, 13, 54-62.

Fillmore, C. J. Review of Bendix's Componential analysis of general vocabulary: The semantic structure of a set of verbs in English. Hindi, and Japanese. International Journal of American Linguistics, 1966, 32, Part II, No. 2, Publication 41.

Fillmore, C. J. Verbs of judging: An exercise in semantic description. In C. J. Fillmore \& D. T. Langendoen (Eds.), Studies in linguistic semantics. New York: Holt, Rinehart \& Winston, 1971.

Fodor, J. A. The language of thought. New York: Crowell, 1975.

Gentner, D. Evidence for the psychological reality of semantic components: The verbs of possession. In D. A. Norman \& D. E. Rumelhart (Eds.), Explorations in cognition. San Francisco: Freeman, 1975.

Gentner, D. On relational meaning: The acquisition of verb meaning. Child Development 1978, 48, 988-998.

Katz, J. J., \& Fodor, J. A. The structure of a semantic theory. Language, 1963, 39 $170-210$.

Kintsch, W. The representation of meaning in memory. Hillsdale, NJ: Erlbaum, 1974.

Kučera, H., \& Francis, W. N. Computational analysis of present-day American English. Providence, RI: Brown University Press, 1967.

Kuczaj, S. A., \& Maratsos, M. P. On the acquisition of front, back and side. Child Development, 1975, 46, 202-210.

Lakoff, G. Irregularity and syntax. New York: Holt, Rinehart and Winston, 1970.

Mandler, G. Organization and memory. In K. W. Spence \& J. A. Spence (Eds.), The psychology of learning and motivation (Volume I). New York: Academic Press, 1967.

McCawley, J. D. The role of semantics in a grammar. In E. Bach \& R. T. Harms (Eds.), Universals in linguistic theory. New York: Holt, Rinehart \& Winston, 1968.

Miller, G. A., \& Johnson-Laird, P. N. Language and perception. Cambridge, MA: Harvard University Press, 1976.

Munro, A. Linguistic theory and the LNR structural representation. In D. A. Norman \& D. E. Rumelhart (Eds.), Explorations in cognition. San Francisco: Freeman, 1975.
Paivio, A. Imagery and verbal processes. New York: Holt, Rinehart \& Winston, 1971.

Reynolds, A. G., \& Flagg, P. W. Recognition memory for elements of sentences. Memory and Cognition, 1976, 4(4), 422-432.

Romney, A. K., \& d'Andrade, R. G. Cognitive aspects of English kin terms. In A. K. Romney \& R. G. d'Andrade (Eds.), Transcultural studies in cognition. American Anthropologist, 1964, 66, (3, Pt. 2), 146-170.

Rumelhart, D. E., \& Levin, J. A. A language comprehension system. In D. A. Norman \& D. E. Rumelhart (Eds.), Explorations in cognition. San Francisco: Freeman, 1975.

Rumelhart, D. E., \& Norman, D. A. The active structural network. In Explorations in cognition. San Francisco: Freeman, 1975.

Rundus, D. Negative effects of using list terms as recall cues. Journal of Verbal Learning and Verbal Behavior, 1973, 12, 43-50.

Schank, R. Conceptual dependency: A theory of natural language understanding. Cognitive Psychology, 1972, 3, 552-631.

Schank, R. C., \& Colby, K. M. (Eds.), Computer models of thought and language. San Francisco: Freeman, 1973.

Slamecka, N. J. An examination of trace storage in free recall. Journal of Experimental Psychology, 1968, 76, 504-513.

Smith, E. E., Shoben, E. J., \& Rips, L. J. Structure and process in semantic memory: A featural model for semantic decisions. Psychological Review, 1974, 81, 214-241.

Thorndyke, P. W. Conceptual complexity and imagery in comprehension and memory. Journal of Verbal Learning and Verbal Behavior, 1975, 14, 359-369.

Tyler, L. K., \& Marsien-Wilson, W. D. The on-line effects of semantic context on syntactic processing. Journal of Verbal Learning and Verbal Behavior, 1977, 16, 683-692.

\section{REFERENCE NOTES}

1. Bowerman, M. The acquisition of word meaning: An investigation of some current conflicts. Paper presented at the Third International Child Language Symposium, London, September 1975.

2. Gentner, D. Towards a psychological theory of the meaning of the possession verbs. Unpublished doctoral dissertation, University of California, San Diego, 1974

3. Gentner, D. Semantic integration at the level of verb meaning. Technical Report No 114, Center for the Study of Reading, 1979.

4. Talmy, L. Semantic structures in English and Atsugewi. Unpublished doctoral dissertation, University of California, Berkeley, 1972.

5. Schank, R., Goldman, N., Rieger, C. J., \& Riesbeck, C. K. Primitive concepts underlying verbs of thought. Computer Science Department, Stanford, Palo Alto, CA STAN-CS-265-72, 1972

(Accepted March 10, 1980) 